\title{
Comunidades vegetales de la costa norte del Golfo San Matías, Río Negro, Argentina
}

\author{
María A. Marcos ${ }^{1,2, \bowtie} \&$ María V. MANCINI ${ }^{1,2}$ \\ 1. Laboratorio de Paleoecología y Palinología. Departamento de Biología. Facultad de Ciencias Exactas y Naturales. UNMDP. \\ Mar del Plata, Pcia. De Buenos Aires. 2. IIMyC-CONICET.
}

\begin{abstract}
RESUMEN. Los ambientes costeros de dunas son ecosistemas complejos con una variación interna elevada y una extensa zonación de la vegetación a lo largo de la línea de costa. La costa norte del Golfo San Matías presenta un sistema de dunas donde la vegetación se distribuye en un gradiente desde la costa. Dicha vegetación está caracterizada por comunidades psammófitas hasta estepas arbustivas xerófitas. Los objetivos de este trabajo fueron 1) describir las principales comunidades vegetales del sitio de estudio, y 2) analizar la asociación entre las comunidades vegetales y las unidades geomorfológicas. Se realizaron 16 censos de vegetación con cuatro réplicas en cada unidad geomorfológica. El análisis de la vegetación realizado permitió describir tres comunidades vegetales. La asociación entre la heterogeneidad florística y las cuatro unidades geomorfológicas de Bajo de la Quinta se vincula principalmente con las características del sustrato.
\end{abstract}

[Palabras clave: vegetación costera, ambiente costero de duna, características geomorfológicas, Monte]

\begin{abstract}
Aвstract. Vegetation communities of north coast of San Matias Gulf, Río Negro, Argentina: Coastal dune environments are complex ecosystems with a high internal variation and extensive zonation of vegetation along the shoreline. The north coast of San Matias Gulf presents a dunes system where the vegetation is distributed in a gradient from the coast with psammophytic communities to the interior with xerophytic shrub communities. The objectives of this article are 1) to describe vegetation communities of the study area, and 2) to analyze the association between communities and geomorphological units. We performed a total of 16 vegetation censuses with four replicates in each geomorphological unit. The vegetation analysis allowed to describe three vegetation communities. The association between floristic diversity and the four geomorphological units of Bajo de la Quinta is mainly linked to the characteristics of the substrate.
\end{abstract}

[Keywords: coast vegetation, dunes coast environments, geomorphological characteristic, Monte]

\section{INTRODUCCIÓN}

La costanorte delGolfoSan Matías se extiende desde la desembocadura del Río Negro hasta el sur de la Bahía San Antonio. El relieve presenta un descenso abrupto, marcado por acantilados de hasta 50 y $60 \mathrm{~m}$ de altura. Esta costa de corte alterna con entrantes costeros correspondientes a bajos topográficos (e.g., el bajo vinculado al desarrollo de Caleta de los Loros-Bahía Creek, el Bajo de la Quinta y el Bajo de San Antonio) que constituyen vías de acceso al mar y donde se desarrollan cordones de gravas, playas arenosas y campos de dunas derivados de estas playas (Favier Dubois \& Borella 2011).

Los ambientes costeros de dunas son ecosistemas complejos con una variación interna elevada y una extensa zonación de la vegetación a lo largo de la línea de costa (Van Der Maarel 1993). En estos sistemas, los factores ambientales como la salinidad, humedad, sustrato y movilidad de la arena son determinantes en el establecimiento

Laboratorio de Paleoecología y Palinología. Departamento de Biología. Facultad de Ciencias Exactas y Naturales. UNMDP. Funes 3250, (7600) Mar del Plata, Pcia. De Buenos Aires. mamarcos@mdp.edu.ar y supervivencia de las comunidades vegetales (van der Maarel 1993). Estos factores ambientales han sido discutidos por Fontana (2005), Monserrat et al. 2006 y Celsi \& Monserrat (2008a y 2008b) en estudios de vegetación costera de dunas del sur de la Provincia de Buenos Aires.

La costa norte del Golfo San Matías presenta un sistema de dunas donde la vegetación se distribuye en un gradiente desde la costa, caracterizada por comunidades psammófitas, hasta el interior con comunidades arbustivas xerófitas características de la provincia biogeográfica del Monte, distrito Sur o Austral (Roig et al. 2009). La vegetación del Monte Austral ha sido abordada en numerosos trabajos (Morello 1958; Cabrera 1971, 1976; León et al. 1998; Roig 1998; Bertiller et al. 2009; Bisigato et al. 2009; Roig et al. 2009; Villagra et al. 2009). La mayoría de los estudios estuvieron enfocados en la vegetación de Monte de Chubut (Bertiller et

\section{Editora asociada: Ana Cingolani}

Recibido: 24 de diciembre de 2011; Fin de arbitraje: 21 de febrero de 2012; Nueva versión: 29 de marzo; tercera versión: 17 de junio; Aceptado: 19 de julio. 
al. 1991; Bisigato \& Bertiller 1997; Bertiller et al. 2004; Bisigato \& Bertiller 2004; Pazos et al. 2010). Pocas descripciones se han realizado en el este de Río Negro (Bran et al. 2007; Kröpfl et al. 2007; Godagnone \& Bran 2009), mientras que la relación entre la vegetación costera y las geoformas presentes en el Golfo San Matías aún no ha sido estudiada. En este sentido, este trabajo aportará información sobre la heterogeneidad de la vegetación en la escala local en el ambiente costero del Golfo San Matías. En particular, como parte de un proyecto de investigación interdisciplinario (arqueológico, geomorfológico y palinológico) en el cual resultó de interés conocer la composición de la vegetación en la localidad de Bajo de la Quinta para reconstruir a partir del análisis polínico la historia de la vegetación durante el Holoceno.

Los objetivos de este trabajo son 1) describir las principales comunidades vegetales del sitio de estudio, y 2) analizar la asociación entre las comunidades vegetales y las unidades geomorfológicas.

\section{MATERIALES y MÉTOdOS}

\section{Área de estudio}

La localidad de Bajo de la Quinta (405'42' S; $64^{\circ} 20^{\prime} 19^{\prime \prime} \mathrm{O}$ ) se encuentra en la costa norte del Golfo San Matías, Río Negro (Figuras 1a y b). Este bajo, de origen fluvial, se halla cubierto parcialmente por un campo de dunas que limitan hacia el este con una laguna (en la actualidad, seca) en la que convergen varias líneas de drenaje (Favier Dubois \& Kokot 2011). Hacia la costa se observa el desarrollo de cordones litorales, flanqueados por acantilados activos al este y al oeste del bajo topográfico. En Bajo de la Quinta, Favier Dubois \& Kokot (2011) diferenciaron cuatro geoformas con mayor extensión: Dunas Móviles, Dunas Fijas, Paisaje Fluvial y Laguna Efímera principalmente (Figura 1c).
Las Dunas Móviles se encuentran representadas por un campo de dunas barjanoides que corren en dirección este-oeste. Son geoformas muy dinámicas, modeladas por la acción eólica que produce modificaciones en períodos cortos de tiempo. Los sectores ocupados por las Dunas Fijas presentan sustratos de mayor estabilidad, asociados a un mayor desarrollo de la vegetación. Esta geoforma se extiende entre las Dunas Móviles y el Paisaje Fluvial en Bajo de la Quinta. Ambas unidades (Dunas Móviles y Fijas) son terrazas marinas cuyas alturas varían entre 10 y 30 m.s.n.m., cubiertas por depósitos arenosos y limo-arenosos, respectivamente. El Paisaje Fluvial es la geoforma más extensa del sitio de estudio, representada por un bajo topográfico que da nombre a la localidad. Estos "bajos" son característicos en la costa norte del Golfo San Matías y representan los entrantes costeros que alternan con acantilados activos a lo largo de la costa. El sustrato limo-arcilloso con mayor contenido de materia orgánica está asociado a condiciones de estabilidad que permite el desarrollo de estepas arbustivas xerófitas. Por último, la unidad geomorfológica Laguna Efímera representa una laguna temporaria pequeña, actualmente seca, cubierta por vegetación graminosa sobre un sustrato limoso. Esta geoforma se encuentra bordeada por la unidad Paisaje Fluvial y presenta drenajes efímeros que abastecen la cuenca como producto de las lluvias esporádicas en la región (Figura 1c).

El clima es templado semiárido, con rasgos bien localizados debido a la influencia del mar. De acuerdo al índice de aridez de Gaussen (1954), el área es de tipo subdesértico, con $~ 270$ días secos (Genchi et al. 2010). La temperatura media anual es de $15{ }^{\circ} \mathrm{C}$ y la precipitación media anual es de $250 \mathrm{~mm}$. Presenta predominancia de los vientos del noroeste entre marzo y noviembre, mientras que en los meses estivales la dirección dominante es sureste (Correa 1998; Labraga \& Villalba 2009; Genchi et al. 2010). La vegetación es de tipo arbustiva, perteneciente a la provincia biogeográfica del Monte, distrito Sur o Austral. A escala regional, la fisonomía es uniforme y está caracterizada por la estepa arbustiva de Larrea spp. (i.e., jarillal) (Cabrera

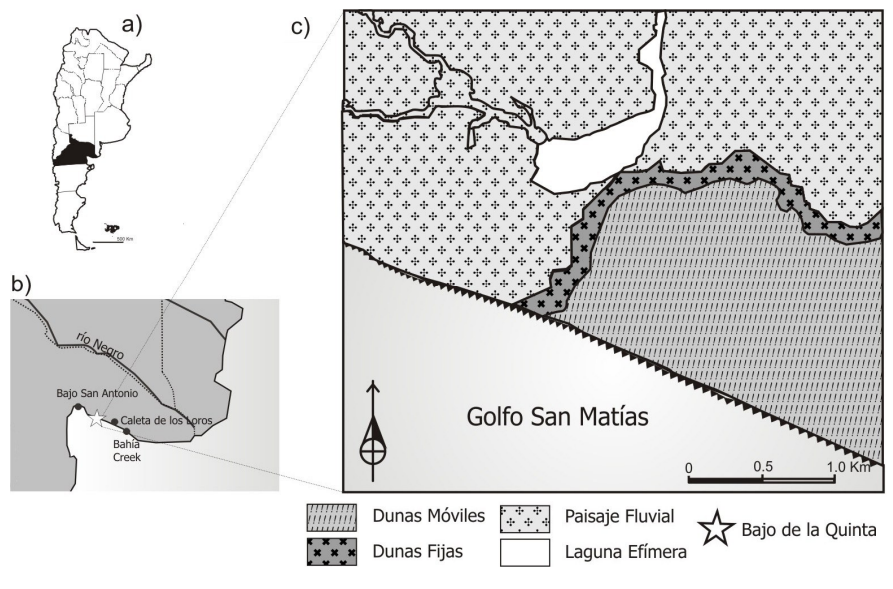

Figura 1. a) Provincia de Río Negro, Argentina, b) Golfo San Matías, Río Negro y ubicación de los bajos topográficos presentes en la costa norte, c) Área de estudio y unidades geomorfológicas de la localidad de Bajo de la Quinta, Río Negro Argentina.

Figure 1. a) Río Negro Province, Argentina, b) San Matías Gulf, Río Negro and topographic lownands location in the north coast, c) Study area and geomorphological units in the Bajo de la Quinta locality, Río Negro Argentina. 
1976; Roig-Juñent et al. 2001; Abraham et al. 2009; Roig et al. 2009). En particular, la vegetación en la costa norte del Golfo San Matías es una estepa arbustiva de varios estratos que oscilan entre 0.5 y $1.5 \mathrm{~m}$. La especie dominante es Larrea divaricata, un arbusto perennifolio de altura inferior a $1.5 \mathrm{~m}$, cuyas hojas presentan caracteres adaptativos a condiciones de altas temperaturas y aridez (León et al. 1998). De acuerdo a Godagnone \& Bran (2009), la estepa arbustiva de Larrea spp. se asocia con otros arbustos, subarbustos y/o con variantes psammófitas y halófitas a lo largo de la costa.

\section{Diseño de muestreo}

Se hicieron un total de 16 censos de vegetación, distribuidos al azar en las cuatro unidades geomorfológicas, con cuatro repeticiones en cada unidad. El área mínima fue estimada de acuerdo a Matteucci \& Colma (1982). Se tomaron muestras en stands de 8x8 m para la vegetación subarbustiva/ arbustiva, y de $4 \times 4 \mathrm{~m}$ para la vegetación herbácea / graminosa. La información recogida en cada censo comprendió la lista de especies de las plantas vasculares identificadas, la altura promedio de los arbustos presentes en cada censo, y la coberturaabundancia total y de cada una de las formas de vida en base a la escala Braun-Blanquet (1979).

Para todos los censos se clasificó la textura del suelo según la estimación táctil hecha a campo en: 1) arenoso, 2) arenoso-arcilloso, 3) arcilloso-limoso, y 4) limoso. Las especies registradas fueron clasificadas según su hábito en arbustos, arbustos y hierbas (Tabla 1) e identificadas de acuerdo a la Flora del Conosur del Darwinion (www2. Darwin.edu.ar/ Proyectos/Flora Argentina/FA.asp).

\section{Análisis de datos}

Para conocer la relación entre los censos de vegetación se realizó un análisis de agrupamiento. Se seleccionó el índice de disimilitud o distancia Euclideana y el método de agrupamiento de Ward (o varianza mínima). De este modo se obtuvo una medida del grado de similitud entre las comunidades vegetales. Este método de agrupamiento determina la varianza existente dentro de los grupos; de esta manera, cada grupo tenderá a ser definido según presente la menor variación posible (Birks \& Gordon 1985; Overpeck et al. 1985). Después se aplicó un análisis de correspondencia destendenciado (DCA) con los datos obtenidos de los censos para comprobar si las diferentes comunidades de vegetación representan a las unidades geomorfológicas presentes. Esta técnica contribuyó a la comprensión de la relación cualitativa entre la vegetación y las geoformas asociadas. La riqueza de especies de cada comunidad vegetal fue calculada como el promedio del número de especies por censo. Se utilizó el índice de Shannon-Wiener $\left(\mathrm{H}^{\prime}=-\sum \mathrm{p}_{\mathrm{i}}\right.$ $\log \mathrm{p}_{\mathrm{i}}$ ) para calcular la diversidad relativa de las especies y la equitatividad (Whittaker 1972; Pielou 1975). En los resultados se expresan los promedios de diversidad, riqueza, equitatividad y cobertura total de arbustos y de hierbas para cada comunidad vegetal. Por otro lado, la variación en la diversidad de especies, riqueza, equitatividad, cobertura total de la vegetación, cobertura de arbustos y cobertura de hierbas en función a las comunidades definidas por el análisis de agrupamiento fue puesta a prueba utilizando un análisis de varianza de una vía (ANOVA) y un test de Tukey (a posteriori) para la comparación múltiple de las medias. Previamente, la normalidad de las variables a analizar fue puesta a prueba mediante el test de Shapiro-Wilk.

\section{Resultados}

\section{Características generales de la vegetación}

En los censos realizados en Bajo de la Quinta se registró un total de 20 especies de plantas vasculares, con una riqueza promedio de 5.2 especies por censo. Las especies más abundantes fueron Larrea divaricata, Capparis atamisquea Kuntze, Nassella tenuis (Phil.) Barkworth, Poa ligularis Phil., Sporobolus rigens (Trin) E. Des. y Hyalis argentea Hook \& Arn. var. Lastisquama Cabrera. Sobre la base de los censos realizados, el análisis de agrupamiento permitió diferenciar tres asociaciones mayores, con una distancia euclidiana de 1.5. Una asociación consiste en censos provenientes de la unidad Laguna Efímera, caracterizada por pocas especies y dominancia de gramíneas. Paisaje Fluvial y Dunas Fijas han sido agrupados en otra asociación con mayor similitud y los más altos valores de riqueza de especies. La última

Tabla1. Clasificación de las especies según su hábito de acuerdo a Flora del Conosur (www2.Darwin.edu.ar/ Proyectos/Flora Argentina/FA.asp).

Table 1. Classification of species based on their habit, according to Flora of the Southern Cone (www2.Darwin.edu.ar/ Proyectos/Flora Argentina/FA.asp).

\begin{tabular}{ll}
\hline Forma de vida & Especies \\
\hline Arbustos perennes & Larrea divaricata \\
& Prosopis alpataco \\
& Capparis atamisquea \\
& Monttea aphylla \\
& Geoffraea decorticans \\
& Cereus aethiops \\
& Condalia microphylla \\
& Chuquiraga erinaceae \\
& Ephedra ochreata \\
& Senecio subulatus \\
& Senna aphylla \\
& Acanthoplippia seriphioides \\
& Hyalis argentea \\
& Grindelia chiloensis \\
& Hoffmanseggia glauca \\
Hierbas nativas & Calycera crassifolia \\
Hierbas perennes & Hydrocotyle ranunculoides \\
& Sporobolus rigens \\
& Nassella tenuis \\
& Poa ligularis \\
\hline
\end{tabular}


asociación consiste en censos de la unidad Dunas Móviles, con los más bajos valores de riqueza de especies. En base a los datos relevados y al análisis de agrupamiento se describen los siguientes tres tipos de vegetación en Bajo de la Quinta (Figura 2; Tabla 2).

Comunidad psammófita. Las especies características de la comunidad están representadas de manera exclusiva por Hyalis argentea y Sporobulus rigens. La contribución de Calycera crassifolia (Miers) Hicken es baja, y esporádicamente se registran ejemplares de Hydrocotyle ranunculoides L.f. Los taxa que vegetan esta comunidad son relativamente escasos. La cobertura vegetal total varía entre 30 y 35\%, siendo frecuentes sectores con ausencia total o casi total de la vegetación. La riqueza promedio presentó los valores más bajos de 3.25 especies.

Estepa arbustiva. La especie más frecuente es Larrea divaricata. Los arbustos acompañantes son Capparis atamisquea, Geoffraea decorticans (Gillies ex Hook \& Arn) Burkart, Prosopis alpataco Phil., Ephedra ochreata Miers., Cereus aethiops Haw., Condalia microphylla Cav., y Monttea aphylla (Miers) Benth. \& Hook., Chuquiraga erinaceae D. Don, Senecio subulatus D. Don ex Hook \& Arn, Grindelia chiloensis (Cornel) Cabrera, con valores de cobertura que varían entre 5 y $50 \%$. Los arbustos enanos están representados por Acantholippia seriphioides (A. Gray) Moldenke (30-50\%) y Senna aphylla (Cav.) H.S. Irwin \& Barneby (25\%). La cobertura de gramíneas (Sporobolus rigens, Poa ligularis y Nassella tenuis) y herbáceas varía entre 10-25\% y $1 \%$, respectivamente. En esta comunidad, el estrato arbustivo adquiere una dominancia elevada. La cobertura total en la comunidad varía entre 30 y $75 \%$, y además tienen lugar los mayores valores de riqueza de especies (7.93).

Estepagraminosa.Poaligularisy Nassellatenuis representan las gramíneas más abundantes de la comunidad. Sus coberturas llegan al 75\%.

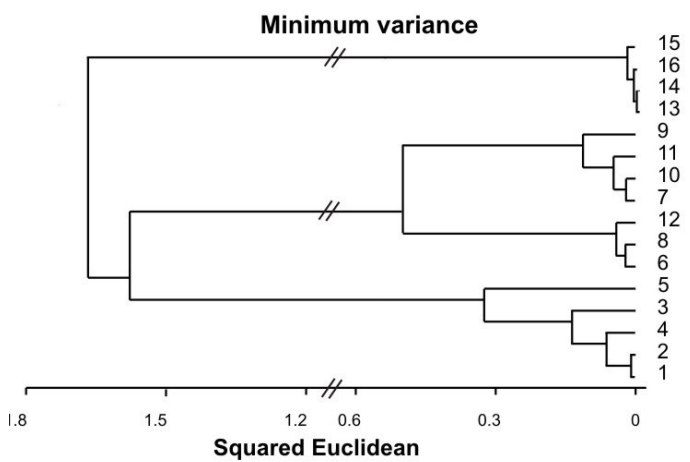

Figura 2. Análisis de agrupamiento de los 16 censos de vegetación de las unidades geomorfológicas estudiadas. Figure 2. Cluster analysis of the 16 vegetation samples studied geomorphological units.

Sin embargo, es notoria la presencia de sectores con parches de suelo desnudo que alternan con el estrato herbáceo/graminoso. El estrato arbustivo está representado escasamente, con ejemplares aislados de Larrea divaricata y Condalia microphylla. La hierba Hoffmanseggia glauca (Ort.) Eifert contribuye a la cobertura total con valores entre 1 y 5\%. El valor medio de la riqueza de especies para la comunidad es 4.625.

Los resultados del análisis de correspondencia destendenciado permiten observar las relaciones entre las unidades geomorfológicas y las comunidades vegetales descriptas (Figura 3). Los primeros dos ejes (autovalores $=0.84 \mathrm{y}$ 0.61 ) explican $26.7 \%$ y $19.51 \%$ de la varianza total, respectivamente. El primer eje ordenó los censos en tres grupos correspondientes a las comunidades vegetales principales del área. Los censos realizados en las Dunas Móviles se agruparon con la comunidad psammófita. Los censos provenientes de las unidades Dunas Fijas y Paisaje Fluvial se reunieron en la comunidad estepa arbustiva, aunque se observa una leve separación espacial entre algunos censos pertenecientes a ambas unidades geomorfológicas. Por último, los censos pertenecientes a la unidad Laguna Efímera se

Tabla 2. Comunidades vegetales, textura del suelo de las unidades geomorfológicas, promedios y análisis de varianza de una vía (ANOVA) entre de riqueza de especies, equitatividad, diversidad, cobertura de hierbas, arbustos y total en función de las tres comunidades vegetales (psammófita, estepa arbustiva y estepa graminosa). Significancia $P<0.05$.

Table 2. Plant communities, soil texture of the geomorphological units, average and analysis of variance (ANOVA) between species richness, evenness, diversity, coverage of grasses, shrubs and total based on the three plant communities (psammophytic, shrub steppe and grass steppe). Significance of $P<0.05$.

\begin{tabular}{lccccc}
\hline Comunidades vegetales & Psammófita & \multicolumn{2}{c}{ Estepa arbustiva } & Estepa graminosa & \multicolumn{2}{c}{ ANOVA } \\
\hline Textura del suelo & Arenoso & Areno-limoso/Limo-arcilloso & Limoso & $\mathrm{F}$ & $\mathrm{p}$ \\
Riqueza de especies & 3.25 & 7.93 & 4.62 & 4.68 & 0.004 \\
Equitatividad & 0.88 & 0.77 & 0.34 & 26.18 & 0 \\
Diversidad & 0.84 & 1.18 & 0.36 & 9.995 & 0.005 \\
Cobertura de arbustos & 13.75 & 50 & 2.5 & 16.79 & 0.001 \\
Cobertura de hierbas & 13.75 & 10 & 68.75 & 70.19 & 0.001 \\
Cobertura total & 28.75 & 60 & 71.25 & 11.73 & 0.003 \\
\hline
\end{tabular}


agruparon respecto a la comunidad vegetal estepa graminosa.

Todas las variables analizadas presentaron una distribución normal (prueba de ShapiroWilk, $P>0.05)$. El análisis ANOVA señaló variaciones significativas en las variables analizadas respecto a las comunidades vegetales (Tabla 2). La comunidad estepa graminosa fue la que más se diferenció del resto de las comunidades. Presentó los menores valores de diversidad y equitatividad respecto a la comunidad psammófita y la estepa arbustiva. Además, mostró valores bajos de cobertura de arbustos respecto a la estepa arbustiva y a la comunidad psammófita. La comunidad psammófita presentó los valores más bajos de riqueza de especies y de cobertura de arbustos en relación a la estepa arbustiva. Además, también se diferenció significativamente en la cobertura total de la vegetación respecto a la estepa arbustiva y la a graminosa.

\section{DisCUSIÓN}

La fisonomía y la composición florística de las comunidades vegetales resultan de procesos bióticos, abióticos, disturbios y restricciones del ambiente (Batista et al. 2005). En los sistemas de dunas costeras, el medio ambiente físico es determinante para el establecimiento y supervivencia de las plantas colonizadoras (van der Maarel 1993). Además, la geomorfología costera juega un rol importante en la zonación de la vegetación (van der Maarel 1993; Fontana 2005). En la localidad Bajo de la Quinta, el análisis de los censos ha permitido relacionar la heterogeneidad florística con las unidades geomorfológicas, y ha señalado una zonación marcada de la vegetación. En particular, la comunidad psammófita y la estepa graminosa presentan una buena correspondencia con las unidades Dunas Móviles y Laguna Efímera, respectivamente. Esta correspondencia se explica por la composición florística característica de cada comunidad. Hyallis argentea y Sporobolus rigens, vegetación asociada a sustratos exclusivamente arenosos (como presentan las Dunas Móviles) son las especies que caracterizan la comunidad psammófita. Por otro lado, Nassella tenuis y Poa ligularis son los representantes dominantes de la comunidad estepa graminosa. Si bien estas especies están presentes en otras geoformas, en particular en esta unidad suceden episodios esporádicos de lluvias que generan pequeñas inundaciones en la laguna. Es posible que esta sea la causa principal por cual no se establece otro tipo de vegetación en esta unidad geomorfológica.

Los censos provenientes de las unidades Dunas Fijas y Paisaje Fluvial se agruparon con la comunidad vegetal estepa arbustiva debido a quecomparten muchas especies (Larrea divaricata, Prosopis alpataco, Acantholippia seriphioides, entre otras). Sin embargo, también existen diferencias en el tipo de sustrato presente en cada unidad geomorfológica, lo cual puede explicar por qué no se observó una separación clara en el DCA entre todos los censos correspondientes a las Dunas Fijas y Paisaje Fluvial.

Si bien la estepa arbustiva de Monte es la comunidad principal del área de estudio, aparece en diferentes contextos geomorfológicos (e.g., bajos, laderas, planicies aluviales, etc.) en la provincia biogeográfica del Monte (Bisigato et al. 2009) asociada a suelos arcillosos y limosos que favorecen su establecimiento. Estos sustratos permiten una mayor disponibilidad de agua, relacionado con la capacidad de infiltración y almacenamiento, que favorece el desarrollo de la vegetación. En el Paisaje Fluvial, estas características del sustrato favorecen el desarrollo de la estepa arbustiva; no obstante, en las Dunas Fijas el sustrato es arenoso-arcilloso, lo que explica la presencia de unos pocos representantes psammófitos en los censos

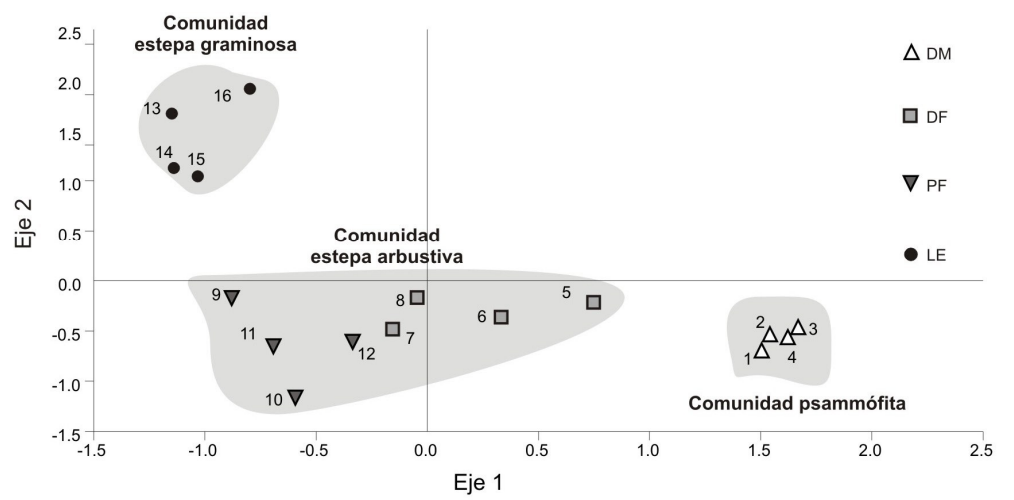

Figura 3. Análisis de correspondencia (DCA) entre los censos de vegetación y las unidades geomorfológicas. DM: Dunas Móviles, DF: Dunas Fijas, PF: Paisaje Fluvial, LE: Laguna Efímera.

Figure 3. Correspondence analysis (DCA) between vegetation samples and geomorphological units. DM: Mobile Dunes, DF: Fixed Dunes, PF: Fluvial Landscape, LE: Ephemeral Ponds. 
realizados y el agrupamiento de los censos 5 y 7 correspondientes a Dunas Fijas en las unidades Dunas Móviles y Paisaje Fluvial en la Figura 2. En este sentido, un gradiente de vegetación zonal entre sitios con suelos arenosos (Dunas Móviles) y sitios con suelos arenoso-arcilloso y arcilloso-limoso (Dunas Fijas y Paisaje Fluvial) es explicado en el eje 1 del análisis de ordenamiento (DCA).

Por otro lado, se exploró la relación entre las unidades geomorfológicas y las comunidades vegetales asociadas en relación a los valores de diversidad, equitatividad, riqueza y cobertura de arbustos, hierbas y total. Las comunidades vegetales con los menores promedios de diversidad y equitatividad fueron la comunidad psammófita y la estepa graminosa respecto a la estepa arbustiva, lo cual puede explicarse por varias causas. En otros estudios de vegetación costera se ha postulado que el tipo de sustrato de las dunas tiene ciertas características (movilidad de la arena, baja capacidad de retención de agua y pobreza de nutrientes) que delimitan conjuntos particulares de especies que pueden establecerse bajo estas condiciones; esto resulta en una diversidad baja (van der Maarel 2005). En este trabajo, la vegetación presente en las Dunas Móviles son dos especies que codominan, Hyalis argentea y Sporobolus rigens, asociadas a áreas de acumulación de arena. En particular, el sustrato arenoso y la baja capacidad de almacenamiento de agua en esta unidad geomorfológica son los factores relevantes en el establecimiento de la vegetación. De acuerdo con Gandullo et al. (1998), las estrategias de colonización de la vegetación psammófita tienen relación con el sistema vegetativo aéreo y con los rizomas que generan una resistencia a la acción del viento produciendo rápidamente la etapa de fijación de la vegetación. Los resultados obtenidos en esta unidad en Bajo de la Quinta son concordantes con otros estudios de vegetación del sur de la costa atlántica bonaerense (Fontana 2005; Monserrat et al. 2006; Celsi \& Monserrat 2008a y 2008b), donde los menores valores de riqueza se registraron en las Dunas Móviles. También hubo correspondencia con las especies psammófitas frecuentes como Hyalis argentea, Sporobolus rigens y Calycera crassifolia entre otras, pertenecientes a las asociaciones de la costa austral descriptas por Cabrera (1941).

Los menores índices de diversidad, equitatividad y cobertura de arbustos en la estepa graminosa son concordantes con datos recolectados durante los censos respecto a la dominancia de gramíneas con grandes parches de suelo desnudo y una presencia escasa de vegetación arbustiva. Las gramíneas de esta unidad presentan estrategias $r$ de colonización, además de una amplia dispersión de semillas y tallos subterráneos rastreros. Esas características, sumadas a la arquitectura de sus raíces, le habrían permitido a las gramíneas hallar condiciones de humedad favorables para su establecimiento (van der Maarel 2005).

Si bien este trabajo representa la primera descripción de la vegetación para la localidad de Bajo de la Quinta, sería interesante considerar en estudios futuros el análisis de otros factores que pueden ser importantes a escala local como el fuego, la herbívora, las interacciones planta-planta, entre otras, que pueden influir en la estructura y composición de las comunidades vegetales.

\section{ConClusiones}

El análisis de la vegetación realizado ha permitido describir tres comunidades vegetales asociadas a las diferentes unidades geomorfológicas de Bajo de la Quinta. La comunidad estepa graminosa presenta una buena correspondencia con la unidad Laguna Efímera. Los episodios esporádicos de lluvias que inundan esta unidad podrían ser la causa principal por la cual no se establece otro tipo de vegetación en el sector. La comunidad estepa arbustiva ha sido asociada tanto en las Dunas Fijas como en el Paisaje Fluvial dado que comparten muchas especies en su composición florística. La comunidad psammófita y la estepa arbustiva señalaron una zonación de la vegetación explicada por el tipo de sustrato (arenoso, arenoso-arcilloso y arcilloso-limoso) presente en las unidades Dunas Móviles, Fijas y Paisaje Fluvial, respectivamente. Este estudio aportó información base de interés para interpretar asociaciones polínicas de secuencias sedimentarias que permiten reconstruir la historia de la vegetación de la costa norte del Golfo San Matías.

Agradecimientos: Los autores agradecen al Dr. Cristian Favier Dubois y la Dra. Florencia Borella directores del proyecto de investigación arqueológica del sitio de estudio, a la Sra. Irma Stabile (dueña de la estancia La Madreselva) y al encargado del campo por permitirnos el acceso al lugar de trabajo. Además, agradecen a los revisores por la lectura crítica y las sugerencias brindadas que han sido de gran ayuda para mejorar el manuscrito. Este estudio fue parte de la Tesis Doctoral de M.A. Marcos y ha sido desarrollado dentro de los siguientes proyectos: PIP CONICET 112-200801-00756, PIP-CONICET 1265 y EXA 510/10 (UNMDP). 


\section{BIBLIOGRAFÍA}

Abraham, E; HF Del Valle; F Roig; L Torres; JO Ares; et AL. 2009. Overview of the geography of the Monte Desert biome (Argentina). J. Arid Environments 73:144-153.

Batista, WB; Taboada, MA; Lavado, RS; SB Perelman \& RJC LEÓN. 2005. Asociación entre comunidades vegetales y suelos en el pastizal de la Pampa Deprimida. Pp. 113127 en: Oesterheld, M; MR Aguiar; CM Ghersa \& JM Paruelo (eds.). La heterogeneidad de la vegetación de los agroecosistemas. Un homenaje a Rolando J.C. León. Facultad de Agronomía. Universidad de Buenos Aires. Pp. 472.

Bertiller, MB; AM BeEsKow \& F CORONATO. 1991. Seasonal environmental variation and plant phenology in arid Patagonia (Argentina). J. Arid Environ. 21:1-11.

Bertiller, MB; AJ Bisigato; Al Carrera \& HF Del Valle. 2004. Estructura de la vegetación y funcionamiento de los ecosistemas del Monte Chubutense. Bol. Soc. Argent. Bot. 39(3-4):139-158.

Bertiller, MB; L Marone; R Baldi \& JO Ares. 2009. Biological interactions at different spatial scales in the Monte desert of Argentina. J. Arid Environ. 73:212-221.

BiRKS, HJB \& ADGORDON. 1985. Numerical methods in Quaternary pollen analysis. Academic Press Inc. London. Pp. 313.

Bisigato, AJ \& MB Bertiller. 1997. Grazing effects on patchy dryland vegetation in northern Patagonia. J. Arid Environ. 36:639-653.

Bisigato, AJ \& MB Bertiller. 2004. Temporal and micro-spatial patterning of seedling establishment. Consequences for patch dynamics in the southern Monte, Argentina. Plant. Ecol. 174:235-246.

Bisigato, AJ; PE Villagra; JO Ares \& BE Rossi. 2009. Vegetation heterogeneity in Monte Desert ecosystems: A multi-scale approach linking patterns and processes. J. Arid Environ.73:182-191.

Bran, DE; GA Cecchi; JJ Gattán; JA Ayesa \& CR López. 2007. Efecto de la severidad del quemado sobre la regeneración de la vegetación en el Monte Austral. Ecología Austral. 17:123-131.

Braun-Blanquet, J. 1979. Fitosociología. Bases para el estudio de las comunidades vegetales. Blume H. (ed.). Pp. 820. Madrid.

CABrera, AL. 1941. Las comunidades vegetales de las dunas costaneras de la Provincia de Buenos Aires. DAGI. Publicaciones técnicas 1(2):5-44.

Cabrera, AL. 1971. Fitogeografía de la República Argentina. Bol. Soc. Argent. Bot. 14:1-42.

CABRERA, AL. 1976. Regiones fitogeográficas argentinas. Enciclopedia argentina de agricultura y jardinería. $\left(2^{\mathrm{a}}\right.$ edición) Tomo II. ACME. Pp 85. Bs. As.

Celsi, CE \& Monserrat, AL. 2008a. La vegetación dunícola en el frente costero de La Pampa Austral (Partido de Coronel Dorrego, Buenos Aires). Multequina 17:73-92.

Celsi, CE \& Monserrat, AL. 2008b. Vascular plants, coastal dunes between Pehuén-có and Monte Hermoso, Buenos Aires, Argentina. Check List 4(1):37-46.

Correa, MN. (ed). 1998. Parte I. Flora Patagónica. Colec. Cient. Inst. Nac. Tecnol. Agropec. Pp 391.

Favier Dubois, CM \& F Borella. 2011. Contrastes en la costa del golfo: una aproximación al estudio del uso humano del litoral rionegrino en el pasado. Capítulo 1. Pp. 13-42 en: Cardillo, M\& F Borella (eds.). Arqueología de pescadores y marisqueadores en Nordpatagonia. Editorial Dunken.

FAVIER DuBOIS, CM \& RR KокOT. 2011. Changing in the Bajo de la Quinta (San Matías Gulf, Northern Patagonia, Argentina): impact of geomorphologic processes in the human use of coastal habitats. Quaternary International 245(1):103-110.
FONTANA, SL. 2005. Coastal dune vegetation and pollen representation in south Buenos Aires Province, Argentina. J. of Biogeog. 32(7):19-35.

GANDULlo, R; J GASTIA \& A BÜNZLI. 1998. Dinamismo de la vegetación psammófila de Senillosa y Plottier Provincia de Neuquén (Argentina). Multequina 7:11-20.

Gaussen, H. 1954. Théori et classification des climats et des microclimats du point de vue Phytogéographique. Actes du VIII Congrés International de Botanique. Paris.

Genchi, SA; ME Carbone; MC Piccolo \& ME Perillo. 2010. Déficit hídrico en San Antonio Oeste, Argentina. Revista de Climatología 10:29-34.

GodAGnONE, R \& D BRAn. 2009. Inventario integrado de los Recursos Naturales de la Provincia de Río negro. Geología, Geomorfología, Suelos, Clima, Vegetación y Fauna. INTA. Pp. 392.

KRÖPfl, AI; VA Deregibus \& GA CeCChI. 2007. Disturbios en una estepa arbustiva del Monte: cambios en la vegetación. Ecología Austral 17:257-268.

Labraga, JC \& Villalba, R. 2009. Climate in the Monte Desert: Past trends, present conditions, and future projections. Journal of Arid Environments 73:154-163.

Leon, RJC; D Bran; M Collantes; JM Paruelo \& A Soriano. 1998. Grandes unidades de vegetación de la Patagonia extra andina. Ecología Austral 8:125-144.

Matteucci, SD \& A Colma. 1982. Metodología para el estudio de la vegetación. Chesneau, EV (ed.). Secretaría General de la Organización de los Estados Americanos Programa Regional de Desarrollo Científico y Tecnológico, Washington, DC.

Monserrat, AL; CE CELSI \& JOCODIGNOTTO. 2006. Zonificación de la vegetación asociada a la geomorfología costera entre Pehuen-có y Monte Hermoso. VI Jornadas Nacionales de Ciencias del Mar, Puerto Madryn. Argentina.

Morello, J. 1958. La Provincia Fitogeográfica del Monte. Opera Lill. 2:1-155.

Overbeck, GE; T Webb \& IC Prentice. 1985. Quantitative Interpretation of Fossil Pollen Spectra: Dissimilarity Coefficients and the Method of Modern Analogs. Quaternary Research 23:87-108.

Pazos, GE; JO Ares \& MB Bertiller. 2010. Quantitative assessment of shrubs-grass mosaic development in grazed shrubblands: An example in the Patagonian Monte (Argentina). J. Arid Environ. 74:998-1002.

PIElou, EC. 1975. Ecological Diversity. New York. Wiley Interscience. USA.

RoIG, F. 1998. La vegetación de la Patagonia. Pp. 48-166 en: Correa, MN (ed.). Flora Patagónica. Colec. Cient. Inst. Nac. Tecnol. Agropec.

ROIG, FA, S ROIG-JUÑENT \& V CORBALÁN. 2009. Biogeography of the Monte Desert. J. Arid Environ. 73:164-172.

Roig-Juñent, S; G Flores; S Claver; G Debandi \& A Marvaldi. 2001. Monte Desert (Argentina): insect biodiversity and natural areas. J. Arid Environ. 47:77-94.

Villagra, PE; GE Defossé; HF Del Valle; S Tanbeni; M ROSTAGNO; ET AL. 2009. Land use and disturbance effects on the dynamics of natural ecosystems of the Monte Desert: Implications for their management. J. Arid Environ. 73:202-211.

Van Der MaArel, E. 1993. Dry Coastal Ecosystems: General Aspects. En: Ecosystem of the world. Vol. 2c. Elservier, Amsterdam.

Van Der MaArel, E. 2005. Vegetation Ecology. Blackwell Publishing, USA. Pp. 395.

WHITTAKER, RH. 1972. Evolution and measurement of species diversity. Taxon. 21:21-251. 